\title{
Recent Advances in Primary Ciliary Dyskinesia: From Diagnosis to Treatment
}

\author{
Ela Erdem Eralp and Bulent Karadag \\ Division of Pediatric Pulmonology, School of Medicine, Marmara University, Istanbul, Turkey
}

$\mathrm{P}$ rimary ciliary dyskinesia (PCD) is a rare inherited disorder of clinical and genetic heterogeneity caused by mutations in approximately 50 known cilia-related genes, with the number of mutations rapidly increasing as research progresses. PCD is a syndrome of several genotypes with overlapping clinical features due to a shared pathology of dysfunctional mucociliary clearance. Diagnosis remains a challenge owing to the lack of symptoms specific to PCD and absence of a gold standard diagnostic test. According to European Respiratory Society and American Thoracic Society guidelines, definitive diagnosis depends on abnormal findings in cilia ultrastructure and/or a positive genetic analysis. These tests require expensive equipment and specialist centres. Besides, there is no therapy specific to PCD and treatment strategies are mainly derived from cystic fibrosis. Early diagnosis and a multidisciplinary approach in clinical follow-up may prevent disease progression and increase quality of life. International collaborations and quality improvement projects are needed to increase the care of patients with PCD.

\section{Keywords}

Primary ciliary dyskinesia, child, nasal nitric oxide, high-speed video-microscopy analysis, transmission electron microscopy, immunofluorescence, genetic analysis, treatment

Disclosures: Ela Erdem Eralp and Bulent Karadag have no financial or non-financial relationships or activities to declare in relation to this article.

Review process: Double-blind peer review.

Compliance with ethics: This study involves a review of the literature and did not involve any studies with human or animal subjects performed by any of the authors.

Data availability: Data sharing is not applicable to this article as no datasets were generated

or analysed during the current study.

Authorship: The named authors meet the International Committee of Medical Journal Editors (ICMJE) criteria for authorship of this manuscript, take responsibility for the integrity of the work as a whole, and have given final approval for the version to be published.

Access: This article is freely accessible at touchRESPIRATORY.com. (C) Touch Medical Media 2021

Received: 3 August 2021

Accepted: 1 November 2021

Published online: 22 December 2021

Citation: touchREVIEWS in Respiratory \&

Pulmonary Diseases. 2021;6(1):14-20

Corresponding author: Ela Erdem Eralp, Pediatric Pulmonology Division, School of Medicine, Marmara Universty, Fevzi Çakmak, Muhsin Yazıcıoğlu Cd No:10, 34899 Pendik, Istanbul, Turkey E: elaerdem@yahoo.com

Support: No funding was received in

the publication of this article.
Primary ciliary dyskinesia (PCD), previously known as immotile cilia syndrome, is a rare genetic disease characterized by abnormal ciliary function due to defects in the structure and/or function of motile cilia, leading to recurrent and chronic infections of the upper and lower airways as a result of impaired mucociliary clearance. ${ }^{1}$

PCD is predominantly inherited in an autosomal recessive pattern, with the exception of FOXJ1, which has an autosomal dominant inheritance, and OFD1 and DNAAF6, which have an X-linked inheritance. ${ }^{2}$ To date, mutations in more than 50 genes have been identified. ${ }^{3}$ These include genes that encode the many proteins that form the cilia axoneme. Most PCD-causing mutations are nonsense, frameshift or splice mutations causing defects in the cilia assembly, structure, function and/or ciliary beating. As a result, abnormal ciliary functions lead to impairment of mucociliary clearance, typically leading to persistent daily wet cough, recurrent sinopulmonary infections, bronchiectasis and serous otitis media. ${ }^{4}$

The prevalence of PCD is estimated to be between 1 in 10,000-20,000 live-born children and is more common in populations with a higher incidence of consanguineous marriage. ${ }^{5,6}$ One of the major problems in PCD is late diagnosis due to nonspecific symptoms, and lack of diagnostic modalities and awareness among physicians. Following international collaborations including registries and researchers from many groups, the high disease burden of PCD is finally being appreciated, with reported increases in diagnoses. ${ }^{-9}$ However, $30 \%$ of patients still cannot be diagnosed despite improvements in diagnostic methods and screening tests..$^{10} \mathrm{Although}$ there is no disease-specific therapy, diagnosis and management by a specialist PCD team is extremely important. This article provides an update on the current data, and reviews the controversies relating to diagnosis and management of PCD based on recent major publications.

\section{Clinical features}

Data on the clinical features of PCD are derived mainly from case series and non-systematic reviews. The symptoms of PCD are nonspecific and reflect motile ciliopathies. The variety and severity of clinical findings are related to the underlying genetic defect. Careful evaluation of clinical features allows the appropriate choice of diagnostic tests. The awareness of PCD among many physicians including paediatricians and otolaryngologists is of great importance, as many common nonspecific findings overlap with other chronic lung diseases such as cystic fibrosis (CF), immune deficiency, asthma and recurrent viral respiratory infections. ${ }^{11}$

PCD has four main clinical features that are important in the differential diagnosis from other respiratory conditions of childhood: laterality defect, unexplained neonatal respiratory distress, early onset and year-round daily nasal rhinosinusitis, and daily wet cough. ${ }^{12}$ Symptoms typically start in infancy. Most children present shortly after birth as term newborns with unexplained respiratory distress manifesting as tachypnoea, prolonged oxygen requirement and upper and 
Figure 1: High-resolution computed tomography of a patient with primary ciliary dyskinesia with dextrocardia showing bronchiectasis in anatomic right middle lobe and lingula

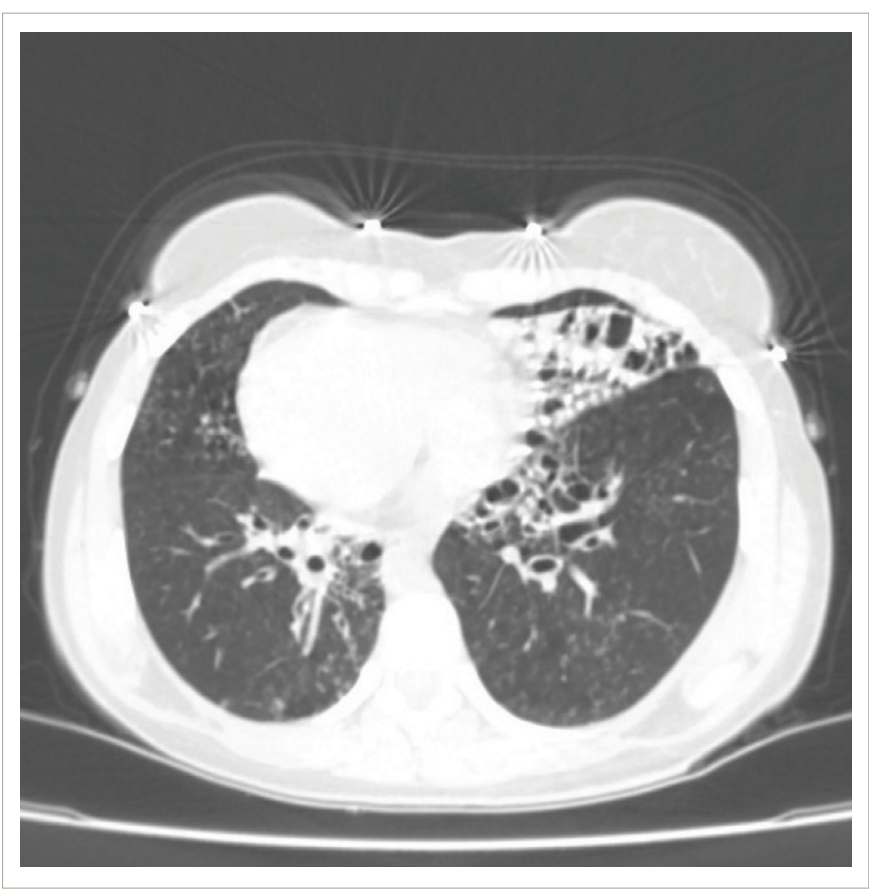

middle lobe atelectasis on chest radiographs. ${ }^{12-14}$ Goutaki et al. reported that $45 \%$ of patients with PCD had a history of neonatal respiratory distress and 30\% were diagnosed during the first 12 months of life in the IPCD cohort, which is the largest dataset of patients with PCD worldwide. ${ }^{15}$ The reduced clearance of amniotic fluid from the neonatal lungs by respiratory cilia is considered a possible explanation for neonatal distress. ${ }^{16}$ Neonatal rhinitis may also be seen and appears hours after birth.

Chronic and recurrent upper respiratory infections develop as a result of decreased mucociliary clearance. Almost $80 \%$ of patients may present with perennial rhinitis and daily, year-round wet cough that begins in early infancy before the age of 6 months. ${ }^{17}$ In the following years, chronic rhinosinusitis, chronic otitis media and ear effusions are seen and may result in conductive hearing loss and speech delay.

Bronchiectasis is also common, often involving the anatomic right middle lobe and lingula (Figure 1). PCD, as the underlying cause of nonCF bronchiectasis, is generally reported more frequently in developed countries. Furthermore, in our recent study, $32.7 \%$ of patients with nonCF bronchiectasis were diagnosed with PCD, compared with $6.3 \%$ in our previous study conducted 20 years ago. ${ }^{18}$

Left-right laterality defects occur in approximately one-half of patients with PCD, and usually manifest as situs inversus totalis, with transposition of the thoracic and abdominal organs as a result of embryonic nodal cilia involvement in a subset of PCD variants (e.g. DNAH5 mutations). ${ }^{19}$ In 10-12\% of the patients, there is heterotaxy associated with congenital heart disease, asplenia and polysplenia. ${ }^{20-22} \mathrm{~A}$ recent meta-analysis found a prevalence of $5 \%$ for congenital heart disease in patients with $P C D{ }^{23}$

Fertility problems (e.g. infertility, subfertility, ectopic pregnancies) due to impaired motility in sperm cells and the fallopian tube, as well as dysmotility of cilia in the efferent duct of the male reproductive tract,
Table 1: Clinical features of primary ciliary dyskinesia

\begin{tabular}{|l|l|}
\hline Neonatal & Unexplained respiratory distress \\
& Situs abnormalities \\
& Congenital heart disease \\
& Other system involvements (e.g. hydrocephalus, \\
& splenic anomalies, hepatic disease) \\
\hline Childhood & Persistent wet cough \\
& Recurrent wheezing \\
& Persistent rhinorrhoea \\
& Chronic rhinosinusitis \\
& Chronic otitis media with effusion \\
& Hearing loss \\
& Recurrent pneumonia \\
& Bronchiectasis \\
\hline Adolescence-adulthood & Chronic rhinosinusitis \\
& Recurrent pneumonia \\
& Bronchiectasis \\
& Fertility problems \\
\hline
\end{tabular}

are another clinical finding. ${ }^{24,25}$ For this reason, genetic counselling to couples pursuing assisted reproductive technology is mandatory. Neonatal hydrocephalus related to dysmotility in brain ventricles is a rare clinical finding in newborns with PCD. It is especially common in patients with reduced generation of multiple motile cilia defects due to CCNO, MCIDAS and FOXJ1 mutations. ${ }^{26,27}$ Primary ciliopathies can affect many different systems depending on the distribution of non-motile cilia in the human body. Accordingly, patients may also present with other system diseases including central nervous malformations, skeletal disorders, psychomotor developmental delay, hepatic and renal diseases, and diabetes, in addition to classical findings of PCD. ${ }^{3,28}$

Therefore, when a patient presents with early onset of recurrent respiratory infections associated with the symptoms presented in Table 1 , it is crucial that they are referred for diagnostic tests for PCD. ${ }^{29}$

\section{Diagnosis}

The approach to PCD diagnosis is still not standardized internationally. A single test is not sufficient and many detailed investigations are required to make the diagnosis. Patients are commonly diagnosed after bronchiectasis and hearing loss. The reasons for underdiagnosis include the rarity of the disease, lack of awareness among physicians, nonspecific nature of symptoms, lack of easy and accessible diagnostic methods, lack of diagnostic tests in many centres and the requirement for expertise in these diagnostic tests, which are also expensive. In addition, there is a lack of standardized diagnostic flow charts and a need for standardization of new techniques. As a result, clinical manifestations continue to play an important role in the diagnosis of PCD.

A valuable clinical tool named PICADAR (Primary Ciliary Dyskinesia Rule) has been developed to predict the probability of PCD. ${ }^{30}$ The PICADAR score is obtained from seven features including: 1) gestation time; 2) neonatal respiratory symptoms; 3) admission in the neonatal period; 4) situs abnormalities; 5) congenital heart defects; 6) persistent year-round rhinitis; 7) chronic otological problems in a child with daily productive cough. Higher scores indicate a higher probability of having PCD. Sensitivity and specificity are 0.90 and 0.75 , respectively, for a cutoff score of $5 .{ }^{30}$ For countries with limited resources, PICADAR could potentially be used to estimate the diagnostic likelihood of patients having PCD; however, the score needs further validation in different settings and populations. ${ }^{31}$ 
The European Respiratory Society (ERS) and American Thoracic Society (ATS) have published guidelines for diagnosis of PCD in order to improve diagnosis. ${ }^{32,33}$ However, there is no single gold standard diagnostic test for a definitive diagnosis. Both guidelines recommend using a combination of investigations, including nasal nitric oxide (nNO), high-speed videomicroscopy analysis (HSVA), transmission electron microscopy (TEM), genetic analysis and immunofluorescence (IF) staining of ciliary proteins.

\section{Nasal nitric oxide}

According to ERS and ATS guidelines, measurement of nNO forms part of the diagnostic work-up. ${ }^{32,33}$ Most individuals with PCD have low nNO levels. NO concentration was first reported to be reduced in the lower airways of children with PCD in 1999. ${ }^{34} \mathrm{NO}$ is thought to play a role in modulating motile cilia function, and NO synthases are localized near basal bodies, which may provide clues about their relationship with motile cilia. ${ }^{35}$ Possible explanations may be an increased breakdown of NO to metabolites, reduced biosynthesis of NO by paranasal sinuses, trapping of $\mathrm{NO}$ in the obstructed paranasal sinuses or reduced production and storage capacity of NO due to agenesis of the sinuses; ${ }^{36}$ however, the exact mechanism remains unclear. Over the age of 6 years, a chemiluminescence analyser with a velum closure technique, which is the gold standard method, is preferred, whereas in children under 6 years of age measurement with tidal breathing is suggested. ${ }^{32}$ The chemiluminescence with velum closure technique has a cut-off value of $77 \mathrm{~nL} / \mathrm{min}$, with $98 \%$ sensitivity and $99 \%$ specificity; ${ }^{37}$ however, diagnostic cut-off values for tidal techniques in preschool children are lacking.

According to the ERS guideline, chemiluminescence analysers can be used as a screening tool, with patients with low nNO values undergoing additional tests to confirm the diagnosis. ${ }^{32}$ Low values can also be seen in $\mathrm{CF}$, nasal polyposis, upper respiratory tract infections and in patients who smoke. It is important to note that patients presenting with a strong clinical history should undergo further testing, even if nNO is normal. Patients with PCD and mutations in GAS8, STK36, CCDC103, DNAH9, RSPH1, NEK10, FOXJ1 and GAS2L2 have normal nNO levels, so measurement of nNO values alone cannot be used as a diagnostic test. ${ }^{38}$

\section{High-speed video-microscopy analysis}

With HSVA, respiratory ciliated cells obtained from the nose or bronchus are examined both with a light microscope and high-speed video camera, and evaluated in terms of number and motility, as well as beat frequency and pattern. Specific beat patterns are classified according to motility as immotile, occasional residual movement, reduced or hyperfrequent with reduced beat amplitude, or with circular motion. ${ }^{28}$ Examination should be performed by keeping the samples at $37^{\circ} \mathrm{C}$ under an inverted microscope. Raidt, et al. found that the normal range was $3.95-8.44 \mathrm{~Hz}$ when measured at $25^{\circ} \mathrm{C} .{ }^{39}$ Recently, Rubbo, et al. reported a $100 \%$ sensitivity and $96 \%$ specificity of HSVA compared with outcomes defined using the ERS guideline. ${ }^{40}$ However, HSVA is a challenging method because the beat frequency is generally affected by infections, nasal drugs, patient age, media used for the maintenance of the sample and the temperature at which the sample is stored. In addition, there is a lack of standardized procedures between centres and a shortage of quantitative data for the interpretation of ciliary beat patterns. Ciliary function also varies under different conditions such as temperature and $\mathrm{pH}$, and a consensus on normative data of temperature among centres should be defined. ${ }^{32}$ Genetic mutations in PCD correlate with ciliary beat pattern variation, and the cilia movement characteristics differ according to the genotype of the patient. ${ }^{39}$ In some mutations (RSPH1, CCDC103, DNAH9, GAS8), cilia movements may appear close to normal, whereas in DNAH11 mutations (normal

\section{Figure 2: Ciliary structure}

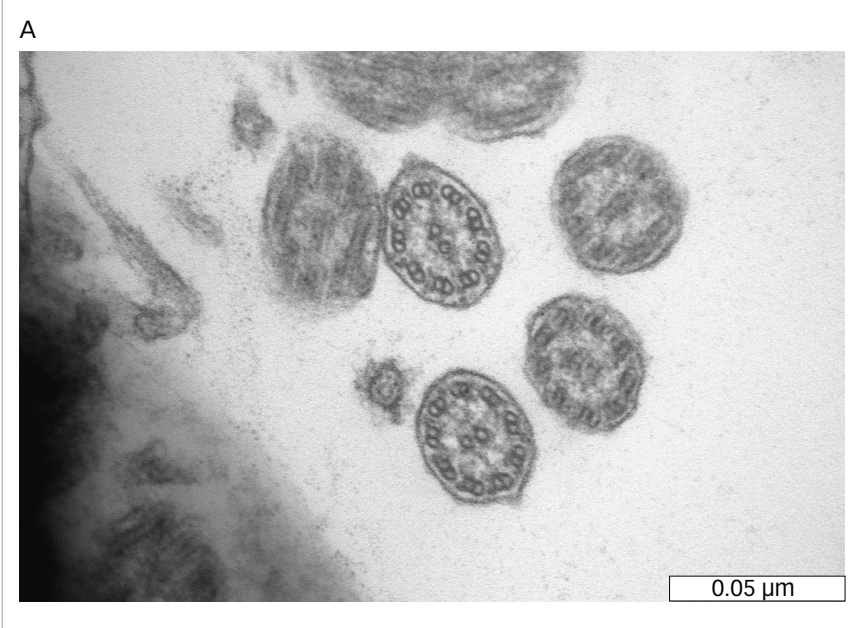

B

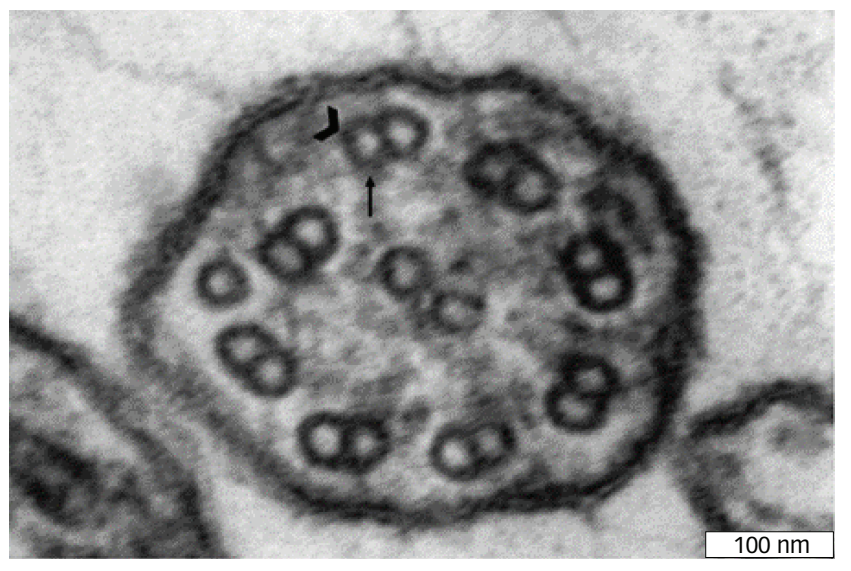

A: Normal ciliary structure. Bars: 0.05 $\mu m ;$ B: Absence of outer (arrowhead) and inner (arrow) dynein arms. Bars: $100 \mathrm{~nm}$

ultrastructure), cilia exhibit a hyperkinetic ciliary beat pattern with reduced proximal axonemal bending. ${ }^{28}$

The ERS recommends using HSVA as part of the diagnostic workup in patients with clinical suspicion of PCD. ${ }^{32}$ Repeated analyses at different times are also recommended to minimize false-positive results; respiratory cell cultures also help to improve the accuracy of HSVA. ${ }^{39}$ HSVA offers a rapid and accurate diagnosis of PCD, although expertise is required to interpret the results.

\section{Transmission electron microscopy}

TEM allows detection of ultrastructural defects by examining transverse sections of cilia (Figure 2). In 33\% of patients with PCD, TEM findings are completely normal, and therefore TEM cannot be used alone to exclude PCD in a patient with strong history. ${ }^{41}$ Besides, only $65-75 \%$ of patients with PCD and positive pathogenic mutations also have pathological TEM findings ${ }^{40}$ TEM is frequently normal or near normal in DNAH11, RPGR and OFD1 mutations, nexin link defects (DRC1,VE, DRC2) and radial spoke defects. ${ }^{28}$ TEM is therefore used in the confirmation of the diagnosis, rather than to rule out $P C D$, because of its high specificity but low sensitivity. In patients with hallmark ciliary ultrastructure defects for PCD, further confirmatory diagnostic investigations are not required. ${ }^{32}$ Findings that definitely suggest the diagnosis of PCD are outer dynein arm defect, outer and inner dynein arm defect, and microtubular disorganization with inner dynein arm defect, also called BEAT PCD TEM Criteria. ${ }^{42}$ Other pathological findings that confirm the diagnosis with other supportive 
evidence are central complex defect, mislocalization of basal bodies, microtubular disorganization defect, outer dynein arm absence, and combined inner and outer dynein arm absence.42

Limitations of TEM include the need for expertise, misdiagnosis due to an inadequate sample, and presence of secondary ultrastructural defects in cilia due to upper and lower respiratory tract infections, tobacco and air pollution exposure or sample handling. ${ }^{42}$ Therefore, samples should be taken when there is no finding of infection, generally $>4$ weeks after an infection.

Both guidelines recommend using TEM in combination with other diagnostic tests, whereas in patients with hallmark findings compatible with $\mathrm{PCD}$, there is no need for further tests. ${ }^{32,33}$

\section{Genetic analysis}

Genetic analysis is recommended in the diagnosis of PCD according to ERS and ATS guidelines. ${ }^{32,33}$ The ATS guideline recommends an extended genetic panel as a diagnostic test over TEM and/or standard ( $>12$ genes) genetic panel testing. ${ }^{33}$ PCD diagnosis is based on having two pathogenic mutations on both alleles of a single gene, defined as biallelic mutation. The majority of reported mutations are nonsense, frameshift or splice mutations, while missense mutations are identified in a minority of cases. Detected mutations should be confirmed by Sanger sequencing, and allele segregation analysis should be performed on the parents and siblings to confirm the genetic diagnosis. It should be kept in mind that large heterozygous genomic deletions that might be missed by DNA sequencing technologies have been reported in PCD individuals. Therefore, next-generation sequencing panel testing is generally preferred for early diagnosis..$^{14}$ Genetic analysis is the diagnostic step in patients with inconclusive results from other diagnostic tests for PCD. However, genetic analysis alone does not exclude the disease in patients with no mutations or in patients with heterozygous variants. Other diagnostic investigations are required to confirm the diagnosis in 30\% of PCD suspected patients whose genetic analysis is not confirmative..$^{28}$

Clinical findings of patients, TEM and HSVA features may vary according to different genetic mutations. Davis, et al. reported that pulmonary involvement in PCD patients with CCDC39 or CCDC40 mutations was more severe compared with patients with mutations effecting dynein arm defects caused by DNAH5 variants. ${ }^{43}$ Conversely, patients with mutations in $R S P H 1$ generally have milder respiratory phenotypes, with later onset of cough and better lung function than other forms of PCD.44 Small case studies have shown that patients with variants in HYDIN, MCIDAS, CCNO, RSPH1, RSPH4A, RSPH9, SPEF2, DRC1 and DRC2 are unlikely to have situs inversus, as nodal cilia are not affected. ${ }^{21,45,46}$ In a recent multinational study, Shoemark, et al. found that disease severity at diagnosis was worse in patients with CCDC39 mutations, better with less reported neonatal respiratory distress in those with DNAH11 mutations and phenotypically diverse in those with DNAH5 mutations. ${ }^{47}$ Unfortunately, uncertainty remains about the differences between genotype and phenotype. It was speculated that some of the patients with pathological variants may not present with typical findings of PCD but may have abnormal cilia motility.

Genetic analysis is an expensive diagnostic test and studies are needed to determine the accuracy. Furthermore, genetic panels must be updated frequently to include all newly discovered PCD-causative genes (Table 2).

\section{Immunofluorescence}

IF microscopy analysis is based on labelling ciliary proteins with fluorescent tags and examining the presence and localization of specific proteins
Table 2: Primary ciliary dyskinesia mutations related to the ciliary structural abnormalities

\begin{tabular}{|c|c|}
\hline Defect on TEM & Gene responsible \\
\hline Outer dynein arm defect & $\begin{array}{l}\text { DNAH1 } \\
\text { DNAH5 } \\
\text { DNAH8 } \\
\text { DNAH9 } \\
\text { DNAI1 } \\
\text { DNAI2 } \\
\text { DNAL1 } \\
\text { NME8 } \\
\text { CCDC103 } \\
\text { ODAD1/CCDC114 } \\
\text { ODAD2/ARMC4 } \\
\text { ODAD3/CCDC151 } \\
\text { ODAD4/TTC25 }\end{array}$ \\
\hline Outer dynein arm and inner arm defect & $\begin{array}{l}\text { DNAAF1 } \\
\text { DNAAF2 } \\
\text { DNAAF3 } \\
\text { DNAAF4 } \\
\text { DNAAF5 } \\
\text { DNAAF6 } \\
\text { SPAG1 } \\
\text { LRRC6 } \\
\text { CFAP298 } \\
\text { CFAP300 } \\
\text { ZMYND10 }\end{array}$ \\
\hline $\begin{array}{l}\text { Inner dynein arm defect with microtubuler } \\
\text { disorganization defect }\end{array}$ & $\begin{array}{l}\text { CCDC39 } \\
\text { CCDC40 } \\
\text { TC12 }\end{array}$ \\
\hline Central pair defect & $\begin{array}{l}\text { HYDIN } \\
\text { SPEF2 } \\
\text { STK36 } \\
\text { CFAP221 }\end{array}$ \\
\hline Nexin dynein regulatory complex defect & $\begin{array}{l}D R C 1 \\
D R C 2 \\
D R C 4 / G A S 8\end{array}$ \\
\hline Radial spoke defect & $\begin{array}{l}\text { DNAJB13 } \\
\text { RSPH1 } \\
\text { RSPH3 } \\
\text { RSPH4A } \\
\text { RSPH9 } \\
\text { NME5 }\end{array}$ \\
\hline Cilia biogenesis defect & $\begin{array}{l}\text { CCNO } \\
\text { MCIDAS } \\
\text { FOXJ1 }\end{array}$ \\
\hline Ciliary base structure & $\begin{array}{l}\text { RPGR } \\
\text { OFD1 } \\
\text { GAS2L2 }\end{array}$ \\
\hline Outer dynein arm proximal defect & DNAH11 \\
\hline Others & $\begin{array}{l}\text { NEK10 } \\
\text { LRRC56 } \\
\text { CFAP57 }\end{array}$ \\
\hline
\end{tabular}

TEM = transmission electron microscopy.

along the cilia by fluorescent or confocal microscopy. IF analysis confims protein absence caused by genetic mutations and helps to demonstrate the effect of the mutation on the cilia. Several antibodies to proteins defective in PCD have been developed and validated, including DNAH5 (an outer dynein arm heavy chain), DNALI1 (an inner dynein arm light chain), 
GAS8 (a component of the nexin-dynein regulatory complex) and RSPH4A RSPH9 and RSPH1 (components of the radial spoke) (Figure 3). ${ }^{1,28,32}$

Recently, it has been shown that PCD variants with normal ultrastructure (e.g. caused by HYDIN and SPEF2 mutations) can be diagnosed by IF. ${ }^{48}$ Shoemark, et al. demonstrated that IF successfully identified 22 of 25 patients with PCD and normal staining in a cohort that was considered highly unlikely to have PCD, as well as providing a result in $55 \%$ of cases that were previously inconclusive. ${ }^{49}$ Genetics and IF findings add much to our understanding of PCD and the relation with TEM findings. For example, in patients with CCNO and DNAH11 mutations, genetic analysis is very important, as IF and TEM findings are generally normal.

The ATS guideline considers IF to be one of the emerging PCD diagnostic techniques, although it has emphasized the lack of consensus on a gold standard for diagnosis and the insufficient sensitivity and specificity when applied to the general population. ${ }^{33}$ The ERS guideline also recommends IF as it is cheaper and easier than the other diagnostic tests and provides a potential test especially in resource-limited settings. ${ }^{32}$

As a result, both ERS and ATS guidelines conclude that there is no single PCD diagnostic test and recommend that a panel of diagnostic tests should be applied to diagnose PCD. The ERS recommends measurement of nNO, TEM, HSVA and genetic analyses, whereas ATS recommends the use of TEM, measurement of nNO and genetic testing. ${ }^{32,33}$ Therefore, diagnosis of PCD depends on at least two of the following methods: typical ciliary ultrastructure defects assessed by TEM, biallelic mutations in PCD-causing genes, abnormal HSVA persisting on at least three repeat tests, abnormal IF examination findings and low nNO values in patients with clinical features suggesting PCD (PICADAR $\geq 5$ ). The availability of diagnostic tests varies between countries. The choice of PCD tests depends on the presence of equipment, experienced laboratories and technical staff.

\section{Pulmonary function tests}

There is heterogeneity in lung function among patients with PCD. Reports of lung function in patients with PCD range from normal to severe impairment. In a recent meta-analysis, it was reported that there was a considerable heterogeneity for both forced expiratory volume in one second (FEV1; 51-96\%) and forced vital capacity (FVC; 66-92\%). ${ }^{50}$ In a study conducted among 991 patients with PCD from the IPCD cohort, mean FEV1 and mean FVC were found to be lower than the mean reference values in all age groups, with best lung function in children aged 6-9 years and the worst in adults. ${ }^{51}$

Lung Clearance Index ( $\mathrm{LCl}$ ) derived from multiple breath washout tests is easier to measure and more sensitive than spirometry in detecting early airway disease in young children. However, there are conflicting data regarding the relationship between FEV1 and $\mathrm{LCl}$ in $\mathrm{PCD}^{52,53}$ In a 1-year follow-up study, Kobbernagel, et al. reported that children and young adults with PCD showed a slight, but significant, increase in LCl with a moderate negative correlation with FEV1. ${ }^{53}$ In another study, Irving, et al. demonstrated that $\mathrm{LCl}$ was more abnormal in patients with microtubular defects than in those with dynein arm defects or normal ultrastructure. ${ }^{54}$ In order to prevent decline in lung function, early standardized care for all patients is very important and prospective studies should be conducted to clarify the role of $\mathrm{LCl}$ in the long-term progression of the disease.

\section{Treatment}

Treatment of PCD requires a multidisciplinary approach including mainly paediatric/adult pulmonologists, otolaryngologists and physiotherapists.
Figure 3: The absence of CCDC39 in respiratory cilia cells from individuals with primary ciliary dyskinesia carrying CCDC39 mutations. Axoneme-specific antibodies against acetylated $\alpha$-tubulin (green) were used as the control. Nuclei were stained with Hoechst 33342 (blue)

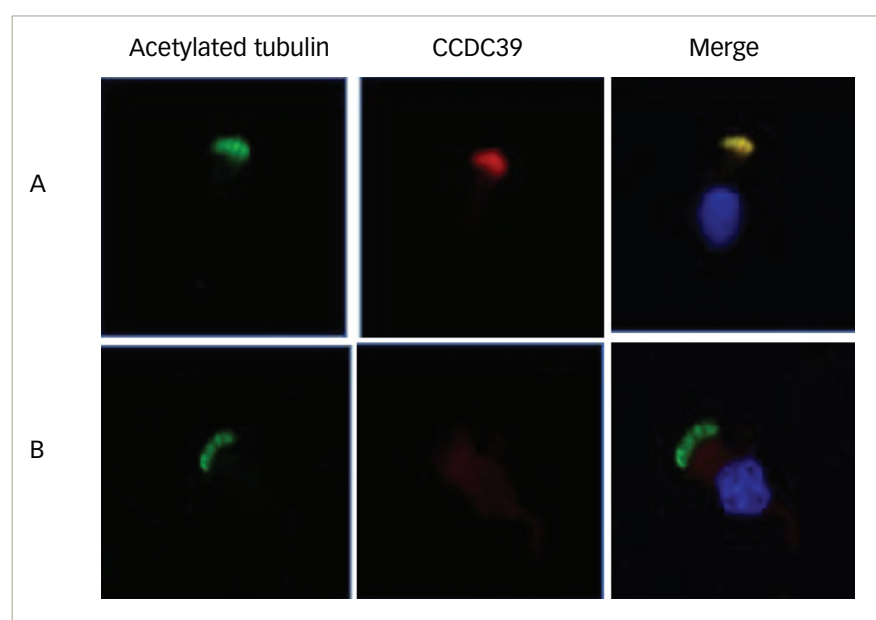

A: In respiratory epithelial cells from healthy probands, CCDC39 (red) localized along the entire length of the axonemes; $B$ : In contrast, in respiratory cilia of individuals with mutations, CCDC39 was absent.

The treatment approach aims to prevent and manage disease complications as there is no effective mutation-specific therapy. Unfortunately, there is a lack of evidence-based treatment for PCD, so early diagnosis followed by initiation of regular physiotherapy and prompt antibiotic treatment in cases of pulmonary exacerbations may prevent lung function impairment and improve long-term outcomes.

\section{Respiratory management}

Given the similarities between CF and PCD, treatment approaches are based on CF management. Mobilization of airway secretions is the main step in PCD management. Many different techniques are available for airway clearance. Conventional chest physiotherapy, including postural drainage, percussion and vibration, is believed to be an effective method in preventing and managing bronchiectasis in PCD. Data comparing different conventional physiotherapy techniques are scarce. Gokdemir, et al. reported a significant increase in lung function with both conventional chest physiotherapy and high-frequency chest wall oscillation in patients with PCD. ${ }^{55}$ In another study comparing conventional chest physiotherapy and oscillatory positive expiratory pressure therapy, both methods were found to be effective techniques in terms of lung function measurement and patient comfort, with patient-reported comfort being higher with oscillatory positive expiratory pressure therapy. ${ }^{56}$ It is therefore appropriate to choose the physiotherapy method according to patient age, compliance and available facilities, and to apply the technique twice daily. Patients should be encouraged to improve aerobic fitness and undertake regular exercise to assist mucus clearance.

Inhaled treatments that alter mucus properties and mucosal hydration may be used to facilitate mucus clearance. In a randomized, controlled study, nebulized $7 \%$ hypertonic saline did not improve quality of life in a small group of adult patients with PCD. ${ }^{57}$ Similarly, there is a lack of evidence on the effect of rhDNase and beta-2 agonists in PCD; currently, only case reports are available showing an improvement in lung function with the use of rhDNase. ${ }^{58}$ Neither of these mucolytic agents therefore are routinely recommended in respiratory management of PCD. Inhaled corticosteroids are also not routinely recommended in PCD and are only used in patient-associated asthma or airway reactivity. 
Early diagnosis and treatment of lower respiratory infections are important to prevent the decline in lung function and bronchiectasis. Pulmonary exacerbation in PCD is defined by the presence of three or more of the following seven items: 1) increased cough; 2) change in sputum volume and/or colour; 3) increased shortness of breath perceived by the patient or parent; 4) decision to start or change antibiotic treatment because of perceived pulmonary symptoms; 5) malaise, tiredness, fatigue or lethargy; 6) new or increased haemoptysis; 7) temperature $>38^{\circ} \mathrm{C} .{ }^{59}$ It is recommended that respiratory tract samples (e.g. sputum, oropharyngeal swabs) are taken regularly every 3-4 months in the follow-up of patients. Streptococcus pneumoniae, Moraxella catarrhalis, Staphylococcus aureus, Haemophilus influenzae and Pseudomonas aeruginosa can be detected in respiratory cultures in children, and $P$. aeruginosa incidence increases with age. ${ }^{43,60} P$. aeruginosa eradication is recommended in $P C D$, but dosing strategies or duration of treatment differ according to centre. ${ }^{3,4,28} \mathrm{~A}$ survey conducted to describe eradication strategies used in European PCD centres revealed that the preferred treatment for first isolation was combined oral ciprofloxacin and inhaled colistimethate sodium in $43 \%$ and nebulized colistimethate for chronic infection with P. aeruginosa in $51 \%$ of the centres. ${ }^{61}$ According to data based on limited studies, appropriate antibiotics should be given for 14-21 days to treat the first isolates of pathogens, and long-term treatment with inhaled antibiotics is recommended for chronic $P$. aeruginosa colonization. ${ }^{62}$ The choice of antibiotic should be based on culture results, when available. In cases of non-response to culture-directed antibiotics, allergic bronchopulmonary aspergillosis testing, non-tuberculous mycobacteria and fungal cultures should be considered.

In patients with frequent pulmonary exacerbations in $\mathrm{CF}$, azithromycin as an anti-inflammatory drug has been shown to reduce the morbidity of exacerbations. In a multicentre study (BESTCILIA), it was demonstrated that twice-weekly maintenance treatment with azithromycin for 6 months in PCD was well tolerated and significantly reduced the rate of exacerbations compared with placebo. ${ }^{63}$

Lung resection to treat PCD lung disease is generally not recommended and is reserved for selected cases with severe and localized bronchiectasis. In a study conducted from data of the IPCD cohort, prevalence of lung resection was found to be $8.9 \%$ among adult patients, and lobectomized patients had lower pulmonary function tests, which declined faster even after surgery compared with nonlobectomized patients. ${ }^{64}$ Non-invasive/invasive ventilation support and lung transplantation should be considered for patients with advanced lung disease and severe respiratory failure. ${ }^{62}$

In terms of upper respiratory tract management, chronic rhinosinusitis can be treated medically with nasal saline irrigations, nasal steroids and antimicrobial agents. ${ }^{28}$ Surgical treatment is indicated in patients who do not respond to medical treatment; endoscopic sinus surgery reduces symptoms and bacterial load in the lower respiratory tract. ${ }^{.5}$ One of the major problems for patients with PCD is the conductive hearing loss due to persistent otitis media with effusion, which may result in delayed speech development, impaired learning and social isolation in the long term. Therefore, effective antibiotic treatment is recommended for acute otitis media. Insufficient data are available for surgical tympanostomy and ventilation tube management. ${ }^{28}$ The ERS guideline recommends hearing aids rather than ventilation tubes, whereas the ATS guideline does not advocate against their use in PCD but recommends counselling on the likelihood of repeated tube insertions after initial placement and on the risk of postoperative otorrhoea. .2, $66^{\circ}$

Sleep-related breathing disorders are common in PCD due to upper airway problems. Oktem, et al. found that patients with PCD have decreased sleep quality and higher rate of sleep disordered breathing compared with controls. ${ }^{67}$

Recently, there has been increased interest in novel therapeutic strategies. Lai, et al. restored DNAH11 gene function ex vivo by replacing the inactivating mutation with wild-type sequence in the diseased cell. ${ }^{68}$ Lee, et al. also described a new IF screening method involving extensive expansion of basal cells from PCD patients and their culture into differentiated respiratory epithelium. The authors concluded that restoration of basal body formation in the patient's nasal epithelial cells would present a novel avenue for drug evaluation and development in PCD. ${ }^{69}$ However, further studies are still needed to investigate other novel treatment approaches for PCD.

\section{Long-term monitoring}

In the long-term follow-up of patients with PCD, monitoring should be done 2-4 times a year, including airway microbiology culture and pulmonary function test by paediatric/adult pulmonologists. For otolaryngology assessment, follow-up is generally recommended 1-2 times a year in children and as needed in adults, as well as audiological examination at the time of diagnosis and annually thereafter, or more often if required. An open-field audiogram is recommended for patients aged $<6$ years and a pure-tone audiogram for patients above this age.

Chest $X$-ray is recommended at the time of diagnosis in cases of pulmonary exacerbations and every 2-4 years in stable patients. Highresolution computed tomography (HRCT) is important in the early diagnosis of bronchiectasis and is recommended at least once after 5-7 years of age when the patient is stable. ${ }^{62}$ However, the use of HRCT in routine follow-up is controversial due to the high risk of radiation exposure. Therefore, magnetic resonance imaging has been increasingly used in recent years. There are no scoring systems specifically for PCD. As differences exist between CF and PCD in terms of structural lung disease, a scoring system specific to PCD is needed. ${ }^{70}$

For prevention of diseases, standard vaccinations according to local schedules, annual influenza vaccine and conjugated pneumococcal vaccines followed by polysaccharide vaccines are recommended. In addition, body weight, height and body mass index should be recorded at each follow-up visit for evaluation of patients' nutritional status.

\section{Conclusion}

PCD is a syndrome with overlapping symptoms and variation in phenotype, according to genotype. This presents many challenges for the diagnosis of PCD, and late rather than early diagnosis is a common problem worldwide. In order to improve the care of patients with $P C D$, there is an urgent need for specialized PCD centres, trained healthcare professionals and international evidence-based therapeutic guidelines. Furthermore, international/national registries should be supported for better and standardized follow-up of patients. Finally, further research to improve diagnostic techniques and identify early biomarkers for lung disease are crucial, together with the development of international guidelines. $\square$ 
1. Werner C, Onnebrink JG, Omran H. Diagnosis and management of primary ciliary dyskinesia. Cilia. 2015;4:2.

2. O'Connor MG, Horani A, Shapiro AJ. Progress in diagnosing primary ciliary dyskinesia: the North American perspective. Diagnostics (Basel). 2021:11:1278.

3. Wallmeier J, Nielsen KG, Kuehni CE, et al. Motile ciliopathies. Nat Rev Dis Primers. 2020;6:77.

4. Rubbo B, Goutaki M, Lucas JS. Primary ciliary dyskinesia. In: Eber E and Midulla F (eds). ERS Handbook Paediatric Respiratory Medicine, 2nd edition. Sheffield, UK: European Respiratory Society, 2021;569-78.

5. O'Callaghan C, Chetcuti P, Moya E. High prevalence of primary ciliary dyskinesia in a British Asian population. Arch Dis Child. 2010;95:51-2.

6. Onoufriadis A, Paff T, Antony D, et al. Splice-site mutations in the axonemal outer dynein arm docking complex gene CCDC114 cause primary ciliary dyskinesia. Am I Hum Genet. 2013;92:88-98.

7. Goutaki M, Maurer E, Halbeisen $\mathrm{FS}$, et al. The internationa primary ciliary dyskinesia cohort (IPCD Cohort): methods and first results. Eur Respir I. 2017:49:1601181.

8. Ardura-Garcia C, Goutaki M, Carr SB, et al. Registries and collaborative studies for primary ciliary dyskinesia in Europe ERJ Open Res. 2020;6:00005-2020.

9. Werner C, Lablans M, Ataian M, et al. An international registry for primary ciliary dyskinesia. Eur Respir J. 2016;47:849-59.

10. Lobo J, Zariwala MA, Noone PG. Primary ciliary dyskinesia. Semin Respir Crit Care Med. 2015;36:169-79.

11. Dehlink E, Hogg C, Carr SB, Bush A. Clinical phenotype and current diagnostic criteria for primary ciliary dyskinesia. Expert Rev Respir Med. 2016;10:1163-75.

12. Leigh MW, Ferkol TW, Davis SD, et al. Clinical features and associated likelihood of primary ciliary dyskinesia in children and adolescents. Ann Am Thor Soc. 2016:13:1305-13.

13. Mullowney T, Manson D, Kim R, et al. Primary ciliary dyskinesia and neonatal respiratory distress. Pediatrics. 2014;134:1160-6.

14. Horani A, Ferkol TW. Advances in the genetics of primary ciliany dyskinesia: clinical implications. Chest. 2018:154:645-52.

15. Goutaki M, Halbeisen FS, Barbato A, et al. Late diagnosis of infants with PCD and neonatal respiratory distress. J Clin Med. 2020;9:2871.

16. Ferkol T, Leigh M. Primary ciliary dyskinesia and newborn respiratory distress. Semin Perinatol. 2006;30:335-40.

17. Sommer JU, Schafer $\mathrm{K}, \mathrm{Omran} \mathrm{H}$, et al. ENT manifestations in patients with primary ciliary dyskinesia: prevalence and significance of otorhinolaryngologic co-morbidities. Eur Arch Otorhinolaryngol. 2011;268:383-8.

18. Eralp EE, Gokdemir $Y$, Atag $E$, et al. Changing clinical characteristics of non-cystic fibrosis bronchiectasis in children. BMC Pulm Med 2020:20:172.

19. Afzelius BA. A human syndrome caused by immotile cilia. Science. 1976;193:317-19.

20. Kennedy MP, Omran H, Leigh MW, et al. Congenital heart disease and other heterotaxic defects in a large cohort of patients with primary ciliary dyskinesia. Circulation. 2007:115:2814-21

21. Best S, Shoemark A, Rubbo B, et al. Risk factors for situs defects and congenital heart disease in primary ciliary dyskinesia. Thorax. 2019;74:203-5.

22. Shapiro AJ, Davis SD, Ferkol T, et al. Laterality defects other than situs inversus totalis in primary ciliary dyskinesia: insights into situs ambiguus and heterotaxy. Chest. 2014;146:1176-86.

23. Goutaki $\mathrm{M}$, Meier $\mathrm{AB}$, Halbeisen $\mathrm{FS}$, et al. Clinical manifestation in primary ciliary dyskinesia: systematic review and metaanalysis. Eur Respir J. 2016:48:1081-95.

24. Vanaken GJ, Bassinet L, Boon M, et al. Infertility in an adult cohort with primary ciliary dyskinesia: phenotype-gene association. Eur Respir J. 2017;50:1700314. Erratum in: Eur Respir I. 2017:50:1750314.

25. Aprea I, Nöthe-Menchen T, Dougherty GW, et al. Motility of efferent duct cilia aids passage of sperm cells through the male reproductive system. Mol Hum Reprod. 2021;27:gaab009.

26. Shapiro AJ, Kaspy K, Daniels MLA, et al. Autosomal dominan variants in FOXJ1 causing primary ciliary dyskinesia in two patients with obstructive hydrocephalus. Mol Genet Genomic
Med. 2021;9:e1726.

27. Robson EA, Dixon $L$, Causon $L$, et al. Hydrocephalus and diffuse choroid plexus hyperplasia in primary ciliary dyskinesia-related MCIDAS mutation Neurol Genet 2020:6:e482.

28. Lucas JS, Davis SD, Omran H, Shoemark A. Primary ciliary dyskinesia in the genomics age. Lancet Respir Med 2020;8:202-16

29. Lucas JS, Paff T, Goggin P, Haarman E. Diagnostic methods in primary ciliary dyskinesia. Paediatr Respir Rev. 2016;18:8-17.

30. Behan L, Dimitrov BD, Kuehni CE, et al. PICADAR: a diagnostic predictive tool for primary ciliary dyskinesia. Eur Respir $J$. 2016;47:1103-12

31. Rumman N, Jackson C, Collins S, et al. Diagnosis of primary ciliary dyskinesia: potential options for resource-limited countries. Eur Respir Rev. 2017;26:160058.

32. Lucas JS, Barbato A, Collins SA, et al. European Respiratory Society guidelines for the diagnosis of primary ciliary dyskinesia. Eur Respir I. 2017:49:1601090.

33. Shapiro AJ, Davis SD, Polineni D, et al. Diagnosis of primary ciliary dyskinesia. An official American Thoracic Society Clinica Practice Guideline. Am J Respir Crit Care Med. 2018:197:e24e39.

34. Karadag B, James AJ, Gültekin E, et al. Nasal and lower airway evel of nitric oxide in children with primary ciliary dyskinesia. Eur Respir J. 1999;13:1402-5.

35. Pifferi M, Bush A, Maggi F, et al. Nasal nitric oxide and nitric xide synthase expression in primary ciliary dyskinesia. Eur Respir J. 2011;37:572-7.

36. Walker WT, Jackson CL, Lackie PM, et al. Nitric oxide in primary ciliary dyskinesia. Eur Respir J. 2012;40:1024-32.

37. Leigh MW, Hazucha MJ, Chawla KK, et al. Genetic disorders of mucociliary clearance consortium. Standardizing nasal nitric oxide measurement as a test for primary ciliary dyskinesia. Ann Am Thorac SOC. 2013:10:574-81.

38. Shapiro AJ, Davis SD, Leigh MW, et al. Limitations of nasal nitric oxide testing in primary ciliary dyskinesia. Am J Respir Crit Care Med. 2020:202:476-7.

39. Raidt J, Wallmeier J, Hjeij R, et al. Ciliary beat pattern and requency in genetic variants of primary ciliary dyskinesia. Eur Respir J. 2014;44:1579-88.

40. Rubbo B, Shoemark A, Jackson CL, et al. Accuracy of highspeed video analysis to diagnose primary ciliary dyskinesia. Chest. 2019;155:1008-17.

41. Boon M, Smits A, Cuppens H, et al. Primary ciliary dyskinesia: critical evaluation of clinical symptoms and diagnosis in patients with normal and abnormal ultrastructure. Orphanet Rare Dis. 2014;9:11.

42. Shoemark A, Boon M, Brochhausen C, et al. International consensus guideline for reporting transmission electron microscopy results in the diagnosis of primary ciliary dyskinesia (BEAT PCD TEM Criteria). Eur Respir J. 2020;55:1900725.

43. Davis SD, Ferkol TW, Rosenfeld M, et al. Clinical features of childhood primary ciliary dyskinesia by genotype and ultrastructural phenotype Am I Respir Crit Care Med. 2015;191:316-24

44. Knowles MR, Ostrowsk LE, Leigh MW, et al. Mutations in RSPH cause primary ciliary dyskinesia with a unique clinical and ciliary phenotype. Am J Respir Crit Care Med. 2014;189:707-17.

45. Olbrich H, Schmidts M, Werner C, et al. Recessive HYDIN mutations cause primary ciliary dyskinesia without randomization of left-right body asymmetry. Am J Hum Genet. 2012;91:672-84

46. Boon $\mathrm{M}$, Wallmeier J, Ma $\mathrm{L}$, et al. MCIDAS mutations result in a mucociliary clearance disorder with reduced generation of multiple motile cilia. Nat Commun 2014:5:4418.

47. Shoemark A, Rubbo B, Legendre M, et al. Topological data analysis reveals genotype-phenotype relationships in primary ciliary dyskinesia. Eur Respir J. 2021;21:2002359.

48 cindric S, Dougherty GW Olbrich H, et al. SPEF2- and HYDINmutant cilia lack the central pair-associated protein SPEF2, aiding primary ciliary dyskinesia diagnostics. Am J Respir Cell Mol Biol. 2020;62:382-96

49. Shoemark A, Frost E, Dixon M, et al. Accuracy of immunofluorescence in the diagnosis of primary ciliary dyskinesia. Am J Respir Crit Care Med. 2017;196:94-101
50. Halbeisen FS, Jose A, de Jong C, et al. Spirometric indices in primary ciliary dyskinesia: systematic review and meta-analysis. . $2019 \cdot 5 \cdot 00231-2018$

51. Halbeisen FS, Goutaki M, Spycher BD, et al. Spirometric indices in primary ciliary dyskinesia: systematic review and metaanalysis. Eur Respir I.2018:52:1801040.

52. Irving S, Carr S, Hogg C, et al. Lung Clearance Index (LCI) is stable in most primary ciliary dyskinesia (PCD) patients managed in a specialist centre: a pilot study. Lung. 2017; 195:441-3.

53. Kobbernagel $\mathrm{HE}$, Green $\mathrm{K}$, Ring AM, et al. One-year evolution and variability in multiple-breath washout indices in children and young adults with primary ciliary dyskinesia. Eur Clin Respir J. $2019 ; 6: 1591841$

54. Irving S, Dixon M, Fassad MR, et al. Primary ciliary dyskinesia due to microtubular defects is associated with worse lung clearance index. Lung. 2018;196:231-8.

55. Gokdemir Y, Karadag-Saygi E, Erdem E, et al. Comparison of conventional pulmonary rehabilitation and high-frequency chest wall oscillation in primary ciliary dyskinesia. Pediatr Pulmonol. 2014:49:611-16.

56. Bingol I, Gokdemir Y, Yilmaz-Yegit C, et al. Comparison of conventional chest physiotherapy and oscillatory positive expiratory pressure therapy in primary ciliary dyskinesia. Pediatr Pulmonol. 2020;55:3414-20.

57. Paff T, Daniels JM, Weersink EJ, et al. A randomised controlled trial on the effect of inhaled hypertonic saline on quality of life in primary ciliary dyskinesia. Eur Respir $J$. 2017:49:1601770.

58. El-Abiad NM, Clifton S, Nasr SZ. Long-term use of nebulized human recombinant DNase1 in two siblings with primary ciliary dyskinesia. Respir Med. 2007;101:2224-6.

59. Lucas JS, Gahleitner F, Amorim A, et al. Pulmonary exacerbations in patients with primary ciliary dyskinesia: an expert consensus definition for use in clinical trials. ERJ Open Res. 2019:5:00147-2018.

60. Alanin MC, Nielsen KG, von Buchwald C, et al. A Iongitudinal study of lung bacterial pathogens in patients with primary ciliary dyskinesia. Clin Microbiol Infect. 2015;21:e1-7.

61. Crowley S, Holgersen MG, Nielsen KG. Variation in treatment strategies for the eradication of Pseudomonas aeruginosa in primary ciliary dyskinesia across European centers. Chron Respir Dis. 2019;16:1479972318787919.

62. Shapiro AJ, Zariwala MA, Ferkol T, et al. Diagnosis, monitoring, and treatment of primary ciliary dyskinesia: PCD foundation consensus recommendations based on state of the art review. Pediatr Pulmonol. 2016;51:115-32.

63. Kobbermagel HE, Buchvald FF, Haarman EG, et al. Efficacy and safety of azithromycin maintenance therapy in primary ciliary dyskinesia (BESTCILIA): a multicenter, double-blind, randomised, placebo-controlled, phase 3 trial. Lancet Respir Med. 2020;8:493-505.

64. Kouis P, Goutaki M, Halbeisen FS, et al. Prevalence and course of disease after lung resection in primary ciliary dyskinesia: a cohort \& nested case-control study. Respir Res. 2019;20:212

65. Alanin MC, Aanaes K, Høiby N, et al. Sinus surgery can improve quality of life, lung infections, and lung function in patients with primary ciliary dyskinesia. Int Forum Allergy Rhinol. 2017;7:240-7.

66. Barbato A, Frischer T, Kuehni CE, et al. Primary ciliary dyskinesia: a consensus statement on diagnostic and treatment approaches in children. Eur Respir J. 2009;34:126476.

67. Oktem S, Karadag B, Erdem E, et al. Sleep disordered breathing in patients with primary ciliary dyskinesia. Pediatr Pulmonol. 2013:48:897-903

68. Lai M, Pifferi M, Bush A, et al. Gene editing of DNAH11 restores normal cilia motility in primary ciliary dyskinesia. $J$ Med Genet 2016:53:242-9

69. Lee DDH, Cardinale D, Nigro E, et al. Higher throughput drug screening for rare respiratory diseases: readthrough therapy in primary ciliary dyskinesia. Eur Respir J. 2021;1:2000455

70. Tadd K, Morgan L, Rosenow T, et al. CF derived scoring systems do not fully describe the range of structural changes seen on CT scans in PCD. Pediatr Pulmonol. 2019;54:471-77. 